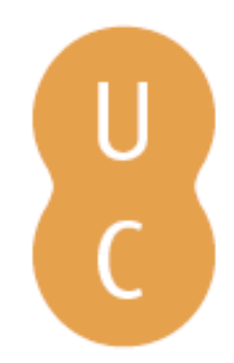

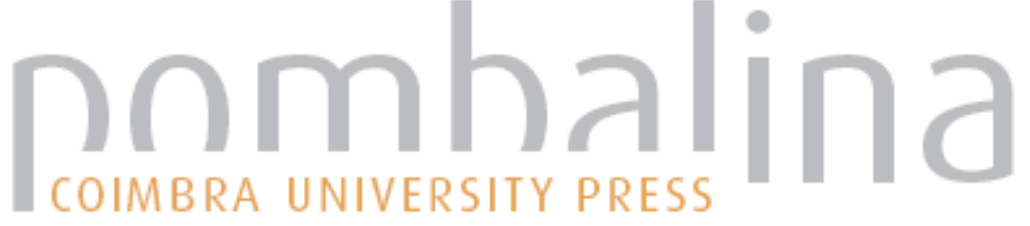

\section{Medicamentos mais utilizados em pediatria e iatrogenia}

Autor(es): Loureiro, Carla Chaves

Publicado por: Imprensa da Universidade de Coimbra

URL

persistente: URI:http://hdl.handle.net/10316.2/43106

DOI: $\quad$ DOl:https://doi.org/10.14195/978-989-26-1300-0_5

Accessed : $\quad$ 26-Apr-2023 10:39:14

A navegação consulta e descarregamento dos títulos inseridos nas Bibliotecas Digitais UC Digitalis, UC Pombalina e UC Impactum, pressupõem a aceitação plena e sem reservas dos Termos e Condições de Uso destas Bibliotecas Digitais, disponíveis em https://digitalis.uc.pt/pt-pt/termos.

Conforme exposto nos referidos Termos e Condições de Uso, o descarregamento de títulos de acesso restrito requer uma licença válida de autorização devendo o utilizador aceder ao(s) documento(s) a partir de um endereço de IP da instituição detentora da supramencionada licença.

Ao utilizador é apenas permitido o descarregamento para uso pessoal, pelo que o emprego do(s) título(s) descarregado(s) para outro fim, designadamente comercial, carece de autorização do respetivo autor ou editor da obra.

Na medida em que todas as obras da UC Digitalis se encontram protegidas pelo Código do Direito de Autor e Direitos Conexos e demais legislação aplicável, toda a cópia, parcial ou total, deste documento, nos casos em que é legalmente admitida, deverá conter ou fazer-se acompanhar por este aviso.

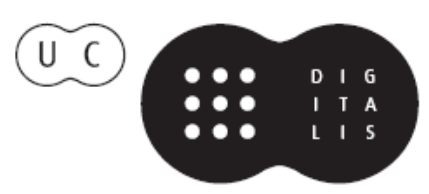


Capítulo 5.

Medicamentos mais utilizados

em pediatria e iatrogenia

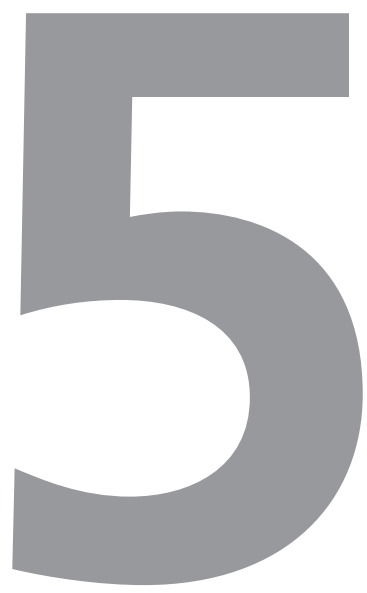

\section{Carla Chaves Loureiro}




\subsection{CONTEXTO}

As crianças pertencem ao grupo dos indivíduos mais vulneráveis da sociedade e a prescrição em idades pediátricas apresenta desafios únicos ao médico.

Os lactentes e as crianças são diferentes dos adultos, não só no tamanho mas também do ponto de vista fisiológico, incluindo a ontogenia da maturidade dos órgãos e a composição corporal, que vão influenciar a ação, eficácia e segurança dos fármacos utilizados.

Por motivos éticos, até 1970, os estudos farmacológicos em crianças, recém-nascidos (RN) e grávidas não estavam recomendados. Assim, a farmacologia pediátrica era extrapolada da prática e experiência em adultos com adaptação das doses - na maioria dos casos com sucesso. Mas, em fármacos com margem terapêutica estreita, como os aminoglicosídeos, por exemplo, os riscos são maiores pelo que requerem um conhecimento mais sofisticado e dosagens individualizadas. Estima-se que 50 a $75 \%$ dos fármacos utilizados em crianças não foram devidamente estudados em populações pediátricas. Em oncologia estes valores atingem $80 \%$ e em neonatologia 90\%.

A utilização de fármacos não aprovados é vulgarmente apelidada de off-label ou off-label drug use (OLDU). A não aprovação pode estar relacionada com a indicação terapêutica, grupo etário, dosagem ou via de administração.

Os problemas resultantes da utilização de fármacos não testados têm sido vários, nalguns casos graves, relacionadas com o fármaco (kernicterus resultante da utilização de sulfizoxazole em RN) ou com o excipiente (o uso de dietileno glicol como solvente ou contaminante de glicerina em várias preparações de xaropes de paracetamol causou mortes em massa na Nigéria, Bangladesh e Haiti).

A inexistência de estudos dirigidos à idade pediátrica deu origem ao aparecimento do termo "órfão terapêutico". Um exemplo de utilização off-label de um fármaco para uma doença órfã é o da aspirina na doença de Kawasaki.

Na década de 70, a Academia Americana de Pediatria argumentou a favor da realização de estudos em crianças: a não realização desses estudos era desprovida de ética por levar os médicos a fazer "experiências" sempre que lhes prescreviam um fármaco e por impedir que as crianças beneficiassem dos seus efeitos farmacoterapêuticos. As primeiras guidelines foram publicadas em 1977 e dois anos depois a Food and Drug Administration (FDA) passou a exigir informação para a idade pediátrica na bula dos medicamentos.

Em 2006, a Europa adotou a Regulamentação Pediátrica da União Europeia (EU) com objetivos legislativos de forma a acelerar e aprofundar a investigação pediátrica. A Comissão Europeia criou o Global Research in Pediatrics (GRiP) de forma a ligar várias redes de trabalho à European Medicines Agency (EMA) permitindo otimizar o desenvolvimento de fármacos pediátricos.

Em 2007 a Assembleia Mundial de Saúde assinou a resolução "Better Medicines for Children" reconhecendo a necessidade de investigação e desenvolvimento de fármacos para as crianças incluindo melhor dosagem, evidência e informação sobre cada medicamento pediátrico. Assim, a Organização Mundial de Saúde (OMS/WHO) desenvolveu uma lista de medicamentos essenciais para crianças e, posteriormente, editou o WHO Model Formulary for Children. 


\subsection{DESCRIÇÃO DO TEMA}

\subsubsection{Uso de fármacos em pediatria}

Para melhor manipular os fármacos em idade pediátrica é fundamental ter alguns conhecimentos relativos a absorção, distribuição, metabolismo e eliminação dos mesmos neste grupo etário.

\subsubsection{Absorção}

\section{Oral/digestiva}

O aparelho gastrointestinal é imaturo no RN e comporta alterações significativas nos primeiros três anos de vida. Até esta idade os níveis de ácido são baixos e os fármacos sensíveis à acidez, como as penicilinas, têm uma absorção aumentada. Por outro lado, fenobarbital, fenitoina e rifampicina têm absorção reduzida - alguns anti-convulsivantes podem ter melhor absorção por via parentérica.

No RN e pequeno lactente, o atraso do esvaziamento gástrico pode ser ultrapassado pela administração endovenosa dos fármacos.

De um modo geral, todos os fármacos devem ser administrados às refeições para diminuir a irritação gástrica e aumentar a compliance - algumas exceções são a isoniazida, a rifampicina, o captopril e as tetraciclinas.

\section{Tópica}

Nas crianças, sobretudo nos lactentes, a absorção tópica está aumentada pelo menor espessamento relativo do estrato córneo, pela maior perfusão cutânea e maior hidratação da epiderme e, ainda, pela relação superior entre a superfície e o peso corporal. Para além disso, a absorção pode ser potenciada numa pele lesada, como no caso das escoriações e das queimaduras, bem como pela utilização de pensos oclusivos. Por exemplo, está totalmente contraindicada a analgesia tópica com lidocaína em crianças (e também em adultos) com queimaduras, pelo risco de morte por bradicardia/paragem cardíaca.

\section{Retal}

A administração de fármacos por via retal pode ser particularmente útil perante doentes com vómito, recusa na ingestão oral, ou quando é necessário jejum. No entanto, esta via não é ideal nem está disponível para muitos fármacos. Para além disso, a variação individual da drenagem venosa retal pode levar a absorção reduzida ou excessiva do medicamento originando concentrações séricas sub-terapêuticas ou tóxicas, respetivamente, pelo que não devem ser administrados, por esta via, fármacos com janela terapêutica estreita. Por outro lado, o risco de expulsão do fármaco, pelas contrações pulsáteis de maior amplitude do reto, não deve ser ignorado.

Em particular, é recomendada a administração retal do diazepam no tratamento de convulsões. O paracetamol também pode ser administrado por via retal mas a absorção pode ser irregular.

\section{Intrapulmonar}

Cada vez mais são utilizados fármacos com efeito no aparelho respiratório por via inalatória com vista a aumentar os efeitos locais e a diminuir a absorção sistémica, nomeadamente broncodilatadores, corticoides e antibióticos. A absorção pulmonar será condicionada pela arquitetura pulmonar e pela capacidade ventilatória mas a investigação, nesta área, está mais centrada na deposição dos fármacos na via aérea do que na absorção pulmonar. 


\subsubsection{Distribuição}

Vários são os fatores que interferem com a distribuição dos medicamentos, entre eles a composição corporal, a afinidade por proteínas plasmáticas e a permeabilidade da barreira hemato-encefálica.

\section{Composição corporal}

A composição corporal das crianças difere da do adulto e varia com o crescimento.

Ao nascimento, $80 \%$ da composição do corpo do RN é água, reduzindo para $65 \%$ aos 12 meses de vida e para $60 \%$ no adulto jovem (homem). Por outro lado, a constituição adiposa varia entre $3 \%$ no prematuro, $12 \%$ no RN de termo, 30\% aos 12 meses e cerca de $18 \%$ no adulto.

Desta forma, os RN e lactentes necessitam de doses superiores $(\mathrm{mg} / \mathrm{kg}$ ) de fármacos hidrossolúveis para atingir concentrações plasmáticas semelhantes às do adulto. No entanto, dada a imaturidade das funções hepática e renal, estes fatores têm de ser considerados para a dose recomendada.

\section{Ligação a proteínas plasmáticas}

No RN, a ligação às proteínas plasmáticas está diminuída pela sua menor concentração plasmática, particularmente a albumina, e há menor capacidade de ligação proteica dos fármacos à albumina fetal. Desta forma vai ocorrer um aumento da fração livre do medicamento farmacologicamente ativo no plasma, com os consequentes incrementos na sua atividade e competição com substâncias endógenas (ácidos gordos livres e bilirrubina), e com outros fármacos com maior afinidade de ligação à albumina.

Contudo, no RN, a maioria das drogas utilizadas tem fraca ligação a proteínas plasmáticas com exceção de fenitoína, sulfonamidas, salicilatos e diazepam, que devem ser administrados com prudência na presença de hiperbilirrubinémia.

Em crianças mais velhas, a ligação a proteínas plasmáticas pode estar alterada, nomeadamente em situações de doença hepática, síndrome nefrótico, insuficiência renal crónica, insuficiência cardíaca e desnutrição.

\section{Barreira hemato-encefálica}

A barreira hemato-encefálica (BHE), que limita a circulação de moléculas para o cérebro, é funcionalmente incompleta no RN pelo que algumas substâncias têm maior penetração encefálica. Para além disso, um dos principais fatores determinantes do transporte de medicamentos através dela é a sua lipossolubilidade, como acontece no caso dos barbitúricos e morfina.

Sendo a meningite e a meningoencefalite doenças mais frequentes na idade pediátrica, a permeabilidade da BHE aos fármacos utilizados é de extrema relevância. É de salientar que, fármacos habitualmente com pouca penetração meníngea podem aumentá-la em situações de inflamação permitindo obter boas concentrações no líquido cefalo-raquídeo (LCR), como penicilinas, cefalosporinas, rifampicina e vancomicina. Medicamentos com boa penetração, mesmo na ausência de inflamação meníngea, incluem o cloranfenicol e o cotrimoxazol. No tratamento de meningites a bactérias Gram-negativas deve-se optar por cefalosporinas, como o cefotaxime, mais apropriadas que os aminoglicosídeos que apresentam uma penetração pouco eficaz e irregular no LCR.

\subsubsection{Metabolismo}

Para além do metabolismo hepático imaturo do RN, também a insuficiência cardíaca e o 
esforço respiratório podem levar a menor atividade metabólica.

O RN, nos primeiros 15 dias de vida, tem um metabolismo baixo que é seguido de um aumento dramático relacionado com as maiores dimensões relativas do fígado na criança. Entre o primeiro e o décimo ano de vida a oxidação microssomal hepática é superior à do adulto determinando que vários fármacos tenham semividas mais curtas na criança (e.g. fenobarbital, fenitoina, teofilina).

\subsubsection{Excreção}

A função renal está imatura no RN de termo e pré-termo mas aumenta significativamente entre o terceiro e o sexto mês de vida atingindo os valores de filtração glomerular do adulto até aos 12 meses.

Avanços recentes na farmacologia clínica pediátrica têm permitido que os fármacos estejam mais bem estudados para cada grupo etário, a posologia seja adequada a cada fase de crescimento e os efeitos secundários melhor estabelecidos. No entanto, muito há ainda a fazer.

Para além da questão da aprovação de um fármaco para crianças, não podemos esquecer outras: será que é mesmo necessário prescrever um fármaco? Que dose vamos utilizar? Com que periodicidade? Durante quanto tempo? Vai interferir com outros fármacos previamente prescritos? E a criança/família vão aderir à terapêutica?

Muitas vezes a resposta está relacionada não com o fármaco em si mas com a doença para que é utilizado (e.g. amoxicilina para amigdalite estreptocócica ou pneumonia pneumocócica, ibuprofeno como antipirético ou anti-inflamatório). A Norma de Orientação Clínica (NOC) - Duração de Terapêutica Antibiótica - demonstra a atenção que está a ser dada à forma de utilização dos fármacos. Outras NOCs têm sido elaboradas especificamente para as crianças como as NOCs - Diagnóstico e Tratamento da Otite Média Aguda, da Amigdalite Aguda e Profilaxia da Endocardite Bacteriana em Idade Pediátrica.

Os esclarecimentos prestados à família são fundamentais na dispensabilidade de prescrição de um fármaco ou no cumprimento de um regime posológico pouco prático. Também algumas dificuldades inerentes à criança devem ser consideradas - se pode ser utilizado, com a mesma eficácia, um fármaco por via oral deve evitar-se uma administração parentérica dolorosa. Mas se a via oral está inviabilizada, por vómitos persistentes ou recusa na sua deglutição, por exemplo, uma via alternativa deve ser obrigatoriamente considerada.

Os fármacos mais utilizados em Pediatria e reanimação neonatal, respetivas vias de administração e doses recomendadas, estão disponíveis para consulta nos quadros 1 e 2 .

\subsection{2 latrogenia}

A iatrogenia é uma das causas de reações adversas a fármacos.

Para além dos vários fatores de risco associados a erros de prescrição e administração de fármacos em geral (erros de prescrição eletrónica ou manual, erros de leitura de rótulo, erros de troca de fármacos...), outros há que aumentam o risco de reações adversas em crianças, especificamente: as diferenças farmacocinéticas e carência de informação específica já abordadas; a necessidade de calcular doses individualizadas de acordo com a idade/peso/superfície corporal/doença específica; a necessidade de preparar 


\begin{tabular}{|c|c|c|c|}
\hline \multirow{2}{*}{\multicolumn{4}{|c|}{\begin{tabular}{|l|l} 
Fármaco & \multicolumn{1}{c}{ Via } \\
Analgésicos, antipiréticos, anti-inflamatórios
\end{tabular}}} \\
\hline & & & \\
\hline Paracetamol & oral, retal & $15 \mathrm{mg} / \mathrm{kg} / \mathrm{dose}, 4 i d$ & $\begin{array}{l}10-20 \mathrm{mg} / \mathrm{kg} / \mathrm{dose} \\
\mathrm{ev}: 15 \mathrm{mg} / \mathrm{kg} / \text { dose, } 4 \text { id }\end{array}$ \\
\hline Ibuprofeno & oral, retal & $5 \mathrm{mg} / \mathrm{kg} /$ dose, 3id & $5-10 \mathrm{mg} / \mathrm{kg} / \mathrm{dose}$ \\
\hline \multicolumn{4}{|l|}{ Antibióticos } \\
\hline Amoxicilina & oral & $50-100 \mathrm{mg} / \mathrm{kg} / \mathrm{d}, 2-3 i d$ & Amigdalite $50 \mathrm{mg} / \mathrm{kg} / \mathrm{d}, 2$ id \\
\hline $\begin{array}{l}\text { Amoxicilina/ácido } \\
\text { clavulânico }\end{array}$ & oral & $\begin{array}{l}4: 1-40-50 \mathrm{mg} / \mathrm{kg}, 2 i d \\
7: 1-50-90 \mathrm{mg} / \mathrm{kg}, 2-3 \mathrm{id}\end{array}$ & $\begin{array}{l}\text { Doses referentes a amoxicilina } \\
\text { ev: } 25-50 \mathrm{mg} / \mathrm{kg} / \text { dose, } 3-4 \mathrm{id}\end{array}$ \\
\hline Azitromicina & oral & $10 \mathrm{mg} / \mathrm{kg}, \mathrm{id}$ & \\
\hline Cefixime & oral & $8 \mathrm{mg} / \mathrm{kg} / \mathrm{d}, 1-2 \mathrm{id}$ & \\
\hline Cefuroxime-axetil & oral & $30-40 \mathrm{mg} / \mathrm{kg} / \mathrm{d}, 2$ id & Máximo 500mg \\
\hline Claritromicina & oral, ev & $15 \mathrm{mg} / \mathrm{kg} / \mathrm{d}, 2$ id & \\
\hline Co-trimoxazol & oral & $\mathrm{SMZ} 40 \mathrm{mg} / \mathrm{kg} / \mathrm{d}, 2$ id & ev: $75-100 \mathrm{mg} / \mathrm{kg} / \mathrm{d}, 4 \mathrm{id}$ \\
\hline Doxiciclina & oral & $4 \mathrm{mg} / \mathrm{kg} 1^{\text {a }}$ dose, $2 \mathrm{mg} / \mathrm{kg}$ id & Brucelose $5 \mathrm{mg} / \mathrm{kg} / \mathrm{d} 1-2$ id \\
\hline Eritromicina & oral & $40-60 \mathrm{mg} / \mathrm{kg} / \mathrm{d}, 2-4 \mathrm{id}$ & \\
\hline Flucloxacilina & oral, ev, im & $50-200 \mathrm{mg} / \mathrm{kg} / \mathrm{d}, 3-4 \mathrm{id}$ & $\begin{array}{l}\text { Administrar 30-60 minutos } \\
\text { antes das refeições }\end{array}$ \\
\hline \begin{tabular}{|l|} 
Nitrofurantoina \\
\end{tabular} & oral & $5-7 \mathrm{mg} / \mathrm{kg} / \mathrm{d}, 4 \mathrm{id}$ & Máximo $400 \mathrm{mg} / \mathrm{d}$, manipulado \\
\hline \begin{tabular}{|l|} 
Trimetropim \\
\end{tabular} & oral & $1-2 \mathrm{mg} / \mathrm{kg} / \mathrm{d}, i d$ & Manipulado \\
\hline \multicolumn{4}{|l|}{ Antifúngicos } \\
\hline Miconazol & oral & $\begin{array}{l}\text { <1mês: } 1-2 \mathrm{ml} / \text { dose, } 2 \text { id } \\
\text { 1M-2A:2,5ml/dose, } 2 \text { id } \\
\text { >2A: } 5 \mathrm{ml} / \text { dose, } 2 \text { id } \\
\text { >6A: } 5 \mathrm{ml} / \text { dose, } 4 \text { id }\end{array}$ & \\
\hline Nistatina & oral & $\begin{array}{l}<3 \mathrm{M}: 1 \mathrm{ml} / \text { dose } \\
\text { 3-12M:2ml/dose } \\
>12 \mathrm{M}: 4-6 \mathrm{ml} / \text { dose }\end{array}$ & $\begin{array}{l}4 \text { id, após refeições } \\
\text { Manter na boca antes de deglutir }\end{array}$ \\
\hline \multicolumn{4}{|l|}{ Anti-helmínticos } \\
\hline Albendazol & oral & >2A: 400mg toma única & Oxíuros: repetir 2 semanas depois \\
\hline Mebendazol & oral & $100 \mathrm{mg} /$ dose, 2 id & Oxíuros: repetir 2 semanas depois \\
\hline Metronidazol & oral, ev & $22,5-45 \mathrm{mg} / \mathrm{kg} / \mathrm{d}, 3 \mathrm{id}$ & Giardia: $40 \mathrm{mg} / \mathrm{kg}$, id, 3 dias \\
\hline Pamoato de pirantel & oral & $10 \mathrm{mg} / \mathrm{kg} / \mathrm{d}$, id & $\begin{array}{l}\text { Oxíuros: repetir } 2 \text { semanas } \\
\text { depois Ascaris: toma única } \\
\text { Ancilostoma: } 3 \text { dias }\end{array}$ \\
\hline Tinidazol & oral & $50-60 \mathrm{mg} / \mathrm{kg} / \mathrm{d}, \mathrm{id}$ & $\begin{array}{l}\text { Giardia e tricomonas: toma única, } \\
\text { repetir } 2 \text { semanas depois }\end{array}$ \\
\hline \multicolumn{4}{|c|}{ Anti-parasitários cutâneos } \\
\hline Benzoato de Benzilo & cutânea & $\begin{array}{l}\text { >2A: aplicar no corpo exceto } \\
\text { cara/cabeça, lavar } 24 \mathrm{~h} \\
\text { depois. Repetir } 3 \text { dias }\end{array}$ & 2-6A: diluição se irritação cutânea \\
\hline Clotrimazol & tópico & Aplicar id & \\
\hline Permetrina $1 \%$ & tópico & $\begin{array}{l}\text { Aplicar } 10 \text { min e lavar, } \\
\text { repetir } 1 \text { semana depois }\end{array}$ & $\begin{array}{l}\text { Escovar com pente metálico } \\
\text { para remover lêndeas }\end{array}$ \\
\hline \multicolumn{4}{|l|}{ Antivíricos } \\
\hline Aciclovir & \begin{tabular}{|l|} 
oral, ev \\
tópico \\
\end{tabular} & Oral: $20 \mathrm{mg} / \mathrm{kg} /$ dose, 4 id & 5 dias na varicela \\
\hline Oseltamivir & oral & $\begin{array}{l}<15 \mathrm{~kg}: 30 \mathrm{mg}, i d, 10 \mathrm{~d} \\
\text { 15-23kg:45mg, id, } 10 \mathrm{~d} \\
\text { 23-40kg:60mg, id, } 10 \mathrm{~d} \\
>40 \mathrm{~kg}: 75 \mathrm{mg}, i d, 10 \mathrm{~d}\end{array}$ & $\begin{array}{l}\text { Profilaxia pós exposição } \\
<1 \text { ano: } 2 \mathrm{mg} / \mathrm{kg}, \text { id, } 10 \mathrm{~d} \\
\text { 1-13A: até } 6 \text { semana }\end{array}$ \\
\hline
\end{tabular}




\begin{tabular}{|c|c|c|c|}
\hline \multicolumn{4}{|l|}{ Anticonvulsivantes } \\
\hline Carbamazepina & oral & $10-30 \mathrm{mg} / \mathrm{kg} / \mathrm{d}$, id & $\begin{array}{l}\text { Iniciar com } 5-10 \mathrm{mg} / \mathrm{kg} \\
\text { Níveis séricos } 4-12 \mathrm{mcg} / \mathrm{ml}\end{array}$ \\
\hline Diazepam & $\begin{array}{l}\text { oral, retal, } \\
\text { ev }\end{array}$ & $\begin{array}{l}0,2-0,3 \mathrm{mg} / \mathrm{kg} / \mathrm{dose}, i d \\
0,5 \mathrm{mg} / \mathrm{kg} / \mathrm{dose}, i d \\
0,1-0,3 \mathrm{mg} / \mathrm{kg} / \text { dose }\end{array}$ & $\begin{array}{r}\text { Retal : }<1 \mathrm{~A}: 2,5 \mathrm{mg} / \text { dose } \\
\text { 1-3A: } 5 \mathrm{mg} / \text { dose } \\
\\
>4 \mathrm{~A}: 5-10 \mathrm{mg} / \text { dose }\end{array}$ \\
\hline Midazolam & $\begin{array}{l}\text { oral, } \\
\text { retal, } \\
\text { nasal, } \\
\text { ev }\end{array}$ & $\begin{array}{l}0,1-0,5 \mathrm{mg} / \mathrm{kg} / \mathrm{dose}, 1-4 i d \\
0,1-0,3 \mathrm{mg} / \mathrm{kg} / \mathrm{dose}, 1-4 \text { id } \\
0,2-0,4 \mathrm{mg} / \mathrm{kg} / \mathrm{dose}, 1-4 \text { id } \\
0,1-0,2 \mathrm{mg} / \mathrm{kg} / \text { dose, } 1-4 \text { id }\end{array}$ & \\
\hline Valproato de sódio & oral & $20-30 \mathrm{mg} / \mathrm{kg} / \mathrm{d}, 2 \mathrm{id}$ & Iniciar com $10-15 \mathrm{mg} / \mathrm{kg}$ \\
\hline \multicolumn{4}{|l|}{ Antihistamínicos } \\
\hline Clemastina & $\begin{array}{l}\text { ev, im } \\
\text { oral }\end{array}$ & $\begin{array}{l}\text { 0,025mg/kg/dose, } 1-2 \text { id } \\
<6 \mathrm{~A}: 1 \mathrm{mg},>6 \mathrm{~A}: 3 \mathrm{mg}\end{array}$ & \\
\hline Desloratadina & oral & $\begin{array}{l}\text { 1-6A: } 1,25 \mathrm{mg}, \text { id } \\
\text { 6-12A: 2,5mg, id } \\
\text { >12A: } 5 \mathrm{mg} \text {, id }\end{array}$ & \\
\hline Ebastina & oral & $\begin{array}{l}\text { 6-12A: } 5 \mathrm{mg}, \text { id } \\
\text { >12A: } 10 \mathrm{mg}, \text { id }\end{array}$ & \\
\hline Hidroxizina & oral & $1-2 \mathrm{mg} / \mathrm{kg} / \mathrm{d}, 2-4 \mathrm{id}$ & Tem efeito sedativo \\
\hline Levocetirizina & oral & $\begin{array}{l}\text { 2-6A:2,5mg/d, } 2 \text { id } \\
>6 \mathrm{~A}: 5 \mathrm{mg} / \mathrm{d}, \text { id }\end{array}$ & \\
\hline Rupatadina & oral & $>6 \mathrm{~A}: 5 \mathrm{mg},>12 \mathrm{~A}: 10 \mathrm{mg}$, id & \\
\hline \multicolumn{4}{|c|}{ Antiácidos, antieméticos, antiulcerosos } \\
\hline Omeprazole & oral & $0,6-0,7 \mathrm{mg} / \mathrm{kg}, 1-2 \mathrm{id}$ & \\
\hline Ranitidina & oral & $>6 \mathrm{M}: 2-4 \mathrm{mg} / \mathrm{kg} / \mathrm{d}, 2-3 \mathrm{id}$ & \\
\hline Sucralfato & oral & $\begin{array}{l}<2 \mathrm{~A}: 250 \mathrm{mg}, \\
2-12 \mathrm{~A}: 500 \mathrm{mg},>12 \mathrm{~A}: 1 \mathrm{~g}\end{array}$ & 3-4 id: antes das refeições \\
\hline \multicolumn{4}{|l|}{ Broncodilatadores } \\
\hline Brometo ipratrópio & neb & $\begin{array}{l}<3 A: 125,3-5 A: 250,>5 A: 500 \\
\text { mcg/dose, } 4-8 \text { id }\end{array}$ & pMDI : 60-120mcg 3-12 id \\
\hline Procaterol & neb & $0.8 \mathrm{mcg} / \mathrm{kg} / \mathrm{dose}, 2-4 \mathrm{id}$ & xarope $(5 \mathrm{mg} / \mathrm{ml}): 0.25 \mathrm{ml} / \mathrm{kg} / \mathrm{dose}$ \\
\hline Salbutamol & $\begin{array}{l}\text { neb } \\
\text { pMDI }\end{array}$ & $\begin{array}{l}75-150 \mathrm{mcg} / \mathrm{kg} / \mathrm{dose} \\
300-800 \mathrm{mcg} / \text { dose }\end{array}$ & 4-6 id, de acordo com necessidade \\
\hline Terbutalina & inal & $1-4 m g, 4-6$ id & de acordo com necessidade \\
\hline \multicolumn{4}{|l|}{ Corticoides } \\
\hline Budesonida & inal & $100-200 \mathrm{mcg}, 1-2$ id & \\
\hline Dexametasona & $\begin{array}{l}\text { oral } \\
\text { ev, im }\end{array}$ & $\begin{array}{l}0.15-0.6 \mathrm{mg} / \mathrm{kg} / \mathrm{dose} \\
0.08-0.3 \mathrm{mg} / \mathrm{kg}, 1-4 \text { id }\end{array}$ & $\begin{array}{l}\text { Laringite estridulosa } \\
\text { Anti-inflamatório }\end{array}$ \\
\hline Fluticasona & inal & $50-200 \mathrm{mcg} / \mathrm{dose}, 1-2$ id & \\
\hline Metilprednisolona & $\begin{array}{l}\text { oral } \\
\text { ev }\end{array}$ & $\begin{array}{l}1-2 \mathrm{mg} / \mathrm{kg} / \mathrm{dose}, 1-2 \text { id } \\
30 \mathrm{mg} / \mathrm{kg}\end{array}$ & Traumatismo medular \\
\hline Prednisolona & oral, ev & $1-2 \mathrm{mg} / \mathrm{kg}, 1-3 \mathrm{id}$ & \\
\hline \multicolumn{4}{|l|}{ Diuréticos } \\
\hline Furosemida & oral & $1-4 \mathrm{mg} / \mathrm{kg}, 1-4 \mathrm{id}$ & ev: $0.5-1 \mathrm{mg} / \mathrm{kg} / \mathrm{dose}, 1-4$ id \\
\hline \multicolumn{4}{|l|}{ Laxantes } \\
\hline Citrato de sódio & retal & 1 id, máximo 3 dias & \\
\hline Glicerina & retal & $1 \mathrm{id}, \mathrm{sos}$ & \\
\hline Lactulose & oral & $\begin{array}{l}<1 \mathrm{~A}: 2.5 \mathrm{ml}, 1-5 \mathrm{~A}: 5 \mathrm{ml} \\
\text { 5-10A:10ml/dose, } 1-2 \text { id }\end{array}$ & \\
\hline Macrogol & oral & $\begin{array}{l}\text { >2A:1saquetas, id } \\
\text { >7A: } 1-2 \text { saquetas, id }\end{array}$ & \\
\hline
\end{tabular}




\begin{tabular}{|c|c|c|c|}
\hline \multicolumn{4}{|l|}{ Vitaminas } \\
\hline Vitamina C & oral & $\begin{array}{l}<6 \mathrm{M}: 25 \mathrm{mg}, \text { id } \\
\text { 7-12M: } 30 \mathrm{mg}, \text { id } \\
\text { 1-8A: } 35 \mathrm{mg}, \text { id } \\
\text { >9A: } 40 \mathrm{mg}, \text { id }\end{array}$ & \\
\hline Vitamina D & oral & $\begin{array}{l}\text { Profilaxia: } \\
\text { 1-12M: 400-600U, id }\end{array}$ & $\begin{array}{l}\text { Doses terapêuticas superiores, } \\
\text { de acordo com patologia }\end{array}$ \\
\hline Polivitamínico & oral & \multicolumn{2}{|c|}{$\begin{array}{l}\text { Protovit N®: } 8 \text { gotas/d (6-10gotas, id) } \\
\text { Dagravit@: Lactentes: 4-8 gotas, crianças: 8-11 gotas, id } \\
\text { Centrum junior@: 4-12A: } 1 \text { comprimido mastigável, id }\end{array}$} \\
\hline \multicolumn{4}{|c|}{ Outros - Reanimação, intoxicações, anemia } \\
\hline Adenosina & ev & $50 \mathrm{mcg} / \mathrm{kg} / \mathrm{dose}$ & Bólus rápido - taquicardia supraventricular \\
\hline Adrenalina & $\begin{array}{l}\text { im } \\
\text { ev } \\
\text { neb }\end{array}$ & $\begin{array}{l}0.01 \mathrm{ml} / \mathrm{kg} / \text { dose }(1: 1000) \\
0.1 \mathrm{ml} / \mathrm{kg} / \text { dose }(1: 10000) \\
1-5 \mathrm{ml} / \text { dose (diluído SF) }\end{array}$ & $\begin{array}{l}\text { Auto injetor de adrenalina: } \\
\text { Anapen } ® / \text { Epipen } ® 0.15 \mathrm{mg} \\
(<25 \mathrm{~kg}) ; 0.3 \mathrm{mg}(>25 \mathrm{~kg})\end{array}$ \\
\hline Ácido acetilsalicílico & oral & $\begin{array}{l}10-15 \mathrm{mg} / \mathrm{kg} / \mathrm{dose}, 1-4 \text { id } \\
75-90 \mathrm{mg} / \mathrm{kg}, 4 \text { id } \\
3-5 \mathrm{mg} / \mathrm{kg}, \text { id }\end{array}$ & $\begin{array}{l}\text { Analgesia } \\
\text { Anti-inflamatório } \\
\text { Anti-agregante plaquetar }\end{array}$ \\
\hline Atropina & ev, im & $10-20 \mathrm{mcg} / \mathrm{kg} / \mathrm{dose}$ & \\
\hline Acetilcisteina & $\begin{array}{l}\text { oral } \\
\text { ev }\end{array}$ & $\begin{array}{l}140 \mathrm{mg} / \mathrm{kg} \\
150 \mathrm{mg} / \mathrm{kg} \text { (doses iniciais) }\end{array}$ & Antidoto do paracetamol \\
\hline Carvão ativado & oral & $\begin{array}{l}<1 \mathrm{~A}: 1 \mathrm{~g} / \mathrm{kg} / \text { dose } \\
1-12 \mathrm{~A}: 1-2 \mathrm{~g} / \mathrm{kg} / \text { dose }\end{array}$ & \\
\hline Fenilefrina $2.5 \mathrm{mg} / \mathrm{ml}$ & nasal & $\begin{array}{l}\text { 1-6A: 1-2gotas, 3-4id } \\
\text { 6-12A: 2-3gotas, 3-4id }\end{array}$ & Máximo 5 dias \\
\hline Ferro & oral & $3-6 \mathrm{mg} / \mathrm{kg} / \mathrm{d}, 2-3 \mathrm{id}$ & Absorção potenciada por vit C \\
\hline Flumazenil & ev & $10 \mathrm{mcg} / \mathrm{kg} / \mathrm{dose}$ & Antagonista de benzodiazepinas \\
\hline Naloxona & ev & $5-10 \mathrm{mcg} / \mathrm{kg} / \mathrm{dose}$ & Antagonista de opiáceos \\
\hline Soluto hidratação oral & oral & $\begin{array}{l}\text { Bioral-suero } \AA \\
\text { Dioralyte } ₫: 1 s q / 200 c c \text { água } \\
\text { Miltina electrolit } \AA\end{array}$ & $\begin{array}{l}\text { Ad libitum } \\
\text { Conservar no frigorífico após } \\
\text { preparação/abertura }\end{array}$ \\
\hline
\end{tabular}

d-dia, cc-cm cúbicos, ev-endovenoso, id-número tomas por dia, im - intramuscular, inal-inalação, neb-nebulização, pMDI-Pressurized Metered Dose Inhaler, sq-saqueta.

Quadro 1. Fármacos em Pediatria, via administração e doses recomendadas.

\begin{tabular}{|l|l|l|l|l|l|}
\hline Fármaco & Concentração desejada & Preparação & $\begin{array}{l}\text { Dose da } \\
\text { preparação }\end{array}$ & Via & $\begin{array}{l}\text { Velocidade } \\
\text { administração }\end{array}$ \\
\hline $\begin{array}{l}\text { Adrenalina } \\
(1: 1000)\end{array}$ & $1: 10000$ & $1 \mathrm{ml}+9 \mathrm{ml} \mathrm{SF}$ & $0.5-1 \mathrm{ml} / \mathrm{kg}$ & ET & Rápida \\
\hline $\begin{array}{l}\text { Adrenalina } \\
(1: 1000)\end{array}$ & $1: 10000$ & $1 \mathrm{ml}+9 \mathrm{ml} \mathrm{SF}$ & $0.1-0.3 \mathrm{ml} / \mathrm{kg}$ & EV & Rápida \\
\hline $\begin{array}{l}\text { Expansor de } \\
\text { volume }\end{array}$ & $\begin{array}{l}\text { SF } 0.9 \%, \text { lactato Ringer, } \\
\text { sangue ORh- }\end{array}$ & - & $10 \mathrm{ml} / \mathrm{kg}$ & EV & $5-10 \mathrm{Minutos}$ \\
\hline Glicose $10 \%$ & - & - & $5 \mathrm{ml} / \mathrm{kg} / \mathrm{dose}$ & EV & \\
\hline Naloxona & $0.4 \mathrm{mg} / \mathrm{ml}$ & $1 \mathrm{ml}$ & $0.1 \mathrm{mg} / \mathrm{kg}$ & Rápida \\
\hline Oxigénio & qb para SpO2 $\geq 92 \%$ & & \\
\hline
\end{tabular}

Quadro 2. Fármacos de reanimação neonatal (preparação e modo de administração). 
manipulados ou diluições com consequentes alterações da estabilidade; compatibilidade e biodisponibilidade; e a necessidade, de instrumentos de medição precisos e de sistemas de administração específicos.

Com frequência, os efeitos iatrogénicos estão relacionados não com o princípio ativo mas com os excipientes.

Alguns destes riscos não se colocam apenas a nível hospitalar mas também no domicílio: facilmente se trocam as doses ou frequência de administração de gotas, xaropes, pomadas...

Vários são os exemplos de iatrogenia com consequências graves: i) o uso prolongado de vasoconstritores nasais pode resultar numa rinite iatrogénica, enquanto o seu uso em doses elevadas pode causar efeitos simpaticomiméticos iatrogénicos; ii) o nimesulide, inibidor seletivo da enzima ciclo-oxigenase-2 com atividade antipirética mais rápida e prolongada que o paracetamol e o ibuprofeno, foi associado a hepatotoxicidade rara mas grave, pelo que o seu uso não é recomendado; iii) o risco de efeitos adversos neurológicos da metoclopramida (sintomas extrapiramidais agudos e discinesia tardia irreversível) é superior nas crianças. Eventos cardiovasculares em crianças com intervalo QT longo determinam uma restrição do uso do fármaco a situações específicas; iv) o síndrome de Reye, caracterizado por encefalopatia aguda não inflamatória e insuficiência hepática gorda degenerativa, resulta da utilização de ácido acetilsalicíco em crianças com doença aguda vírica, respiratória ou digestiva.

\subsection{FACTOS A RETER}

Numa criança deve ser bem ponderada a necessidade de farmacoterapia. A utilização de fármacos com efeito placebo deve ser excecional.
Nas patologias que necessitam de terapêutica farmacológica, a posologia deve ser adaptada à idade da criança e à doença. Deve, sempre, ser ponderado o risco/benefício da utilização, ou não, de cada tratamento. E às vezes (muitas vezes) esperar sem intervir também é tratar.

A prescrição deve ser acompanhada de explicação oral e escrita de forma a evitar erros.

Sempre que uma prescrição inclua um dispositivo terapêutico deve ser feito o ensino ou revisão da técnica de utilização do mesmo.

Atualmente, a investigação farmacológica em crianças tem aumentado mas ainda apresenta muitos desafios e, em muitas situações, permanecem órfãos terapêuticos.

\section{Leitura complementar}

Wittich CM, Burkle CM, Lanier WL. Ten common questions (and their answers) about off-label drug use. Mayo Clin Proc 2012;87(10)982-90

Walter DC. Off-label and unlicensed prescribing for children: have we made any progress? Br J Clin Pharmacol 2007; 64(1): $1-2$

WHO Model Formulary for Children 2010. www.who.int/ selection_medicines/list/WMFc

Anjos R, Bandeira T, Marques JG. Formulário de Pediatria. Lisboa 2004

Levine SR et al. Guidelines for preventing medication errors in pediatrics. J Pediatr Pharmacol Ther 2001;6:426-42

Turner MA, Catapana M, Hirschfeld S, Giaquinto C. Pediatric drug development: The impact of evolving regulations. Adv Drug Delivery Rev 2014;73:2-13 